\title{
Über die Einwirkung von Kupfer auf Natriumnitrit.
}

\author{
Von Walter Peters.
}

Da das Reduktionspotential des Kupfers in wäBriger Lösung noch nicht genügend erforscht ist, war es von Interesse, die Einwirkung von metallischem Kupfer auf eine kohlensäurehaltige Lösung von Natriumnitrit beim Erwärmen zu studieren.

Es stellte sich heraus, dab keine katalytische, sondern eine chemische Zersetzung des Salzes stattfand. Das Nitrit bleibt teils unverändert, teils wird es zu Stickstoffoxyd und Stickstoffoxydul reduziert, teils durch den dabei freigewordenen Sauerstoff zu Nitrat oxydiert.

Kis gelang mir jedoch nicht, die Reaktion in eine chemische Gleichung zu bringen. - Versuche, bei denen ich andere Metalle auf salpetrigsaures Natron einwirken lieB, führten zu keiner Gaseutwicklung. Das Kupfer hat also bezüglich der Einwirkung auf Nitrite unter den Metallen nicht seinesgleichen.

\section{Versuche.}

Zündhütchen aus Kupfer wurden in einer Porzellanschale geglübt, um anhaftenden Staub zu verbrennen. Darauf wurden sie in einem Verbrennungsrohre in Wasserstoffstrome reduziert. Für die Reaktion wurde immer auf 2 Atome Kupfer 1 Mol. Natriumnitrit genommen. Von letzterem wurde eine $5 \%$ ige wäbrige Lösung hergestellt.

Zuerst leitete ich durch diese Lösung Kohlendioxyd, um die vom Wasser absorbierte Luft auszutreiben. Danach schüttete ich das Kupfer hinein und verdrängte alle Luft aus dem Kolben durch Kohlensäure. - Inzwischen schwärzte das Nutrit bereits das Kupfer; Gasblasen bildeten sich an ihm und stiegen von ihm auf.

Nunmehr setzte ich den Fraktionierkolben auf das Wasserbad, um die Lösung auf ca. $60^{\circ}$ zu erwärmen, verband das Ansatzrohr mit einem Stickstofisammler, der konzentrierte Kalilauge enthielt und fing das von der Zersetzung des Nitrits herrührende Gas auf.

Letzteres kam danach in eine Eisenvitriol- und in eine Alkoholpipette, um Stickoxyd bzw. Stickstoffoxydul zur Absorptiou zu bringen und auf diesem Wege zu messen. 
Die salpetrige Säure wurde bestimmt:

a) Auf kolorimetrischem Wege, mittels Vergleichs der durch Sulfanilsäure und Naphthylamin rotgefärbten Zersetzungsflüssigkeit mit einer durch dieselben Reagenzien rotgefärbten Lösung von bekanntem Gehalte an Natriumnitrit;

b) auf maßanalytischem Wege, durch Titrieren der Zersetzungsflüssigkeit mit $1 / 2-n-$ Kaliumpermanganat.

Die Salpetersäure wurde in Versuchslösung, die im Fraktionierkolben zurückgeblieben war, nach der Methode von ScrulzeTiemann bestimmt, jedoch die Luft aus dem die Ferrochloridlösang enthaltenden Kolben nicht durch Wasserdampf, sondern durch Kohlendioxyd verdrängt.

I. $59.5 \mathrm{~g}$ (2 Atome) Kupfer, $32.5 \mathrm{~g}$ (1 Mol.) Natriumnitrit: $62.4 \mathrm{ccm}$ NO in $120.0 \mathrm{ccm}$ Gesamtgas.

II. $57.2 \mathrm{~g}$ (2 Atome) Kupfer, $31.3 \mathrm{~g}(1 \mathrm{Mol}$ ) Natriumnitrit: $56.5 \mathrm{ccm}$ NO in $114,9 \mathrm{ccm}$ Gesamtgas.

III. $43.8 \mathrm{~g}$ (10 Atome) Kupfer, $4.8 \mathrm{~g}$ ( 1 Mol.) Natriumnitrit: $95.9 \mathrm{~cm} \mathrm{NO}, 2.7 \mathrm{~cm} \mathrm{~N} \mathrm{~N}_{2} \mathrm{O}$ in $120.2 \mathrm{ccm}$ Gesamtgas.

$\mathrm{HNO}_{2}$-Bestimmung auf kolorimetrischem Wege:

$0.0833 \mathrm{~g} \mathrm{NaNO}_{2}$ wurden in $1 / 21 \mathrm{H}_{2} \mathrm{O}$ gelöst. $-1 \mathrm{ccm}$ dieser

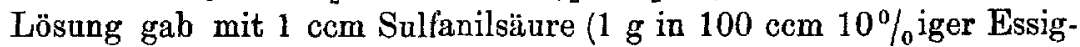
säure) und $1 \mathrm{ccm} \alpha$-Naphthylaminlösung dieselbe Rotfärbung wie $5 \mathrm{ccm}$ von den $134 \mathrm{ccm}$ Zersetzungs甘lüssigkeit in 20000 facher Verdünnung. Letztere enthielt also $4.465 \mathrm{~g} \mathrm{NaNO}_{2}$.

$$
\mathrm{HNO}_{3} \text {-Bestimmung: }
$$

$44.0 \mathrm{ccm}$ der Lösung ergaben $77.5 \mathrm{ccm} \mathrm{N}$ bei $19^{\circ}$ und $757.5 \mathrm{~mm}$ nach zweitägigem Stehen. Also enthielten die $134 \mathrm{ccm} 0.835 \mathrm{NaNO}_{3}$.

IV. $49 \mathrm{~g}$ (10 Atome) Kupfer, $5.4 \mathrm{~g}$ (1 Mol.) Natriumnitrit: $131.2 \mathrm{ccm} \mathrm{NO}, 20.2 \mathrm{ccm} \mathrm{N}_{2} \mathrm{O}$ in $169.0 \mathrm{ccm}$ Gesamtgas.

$\mathrm{HNO}_{2}$-Bestimmung: a) Auf maBanalytischem Wege:

$20 \mathrm{ccm}$ von den erhaltenen $98 \mathrm{ccm}$ Zersetzungsflüssigkeit wurden auf $100 \mathrm{ccm}$ verdünnt, von denen $52.5 \mathrm{ccm} 20.0 \mathrm{ccm} 1 / 2-\mathrm{n}-\mathrm{KMnO}_{4}$ entfärbten. - Demnach enthielten die erwähnten $98 \mathrm{ccm} 3.38 \mathrm{~g} \mathrm{NaNO}_{2}$.

b) Auf kolorimetrischem Wege:

$0.1014 \mathrm{~g} \mathrm{NaNO}_{2}$ wurden in $11 \mathrm{H}_{2} \mathrm{O}$ gelöst und $7.0 \mathrm{~cm}$ dieses Liters auf $100 \mathrm{ccm}$ verdünnt. Diese Lösung wies, mit je $1 \mathrm{ccm}$ meiner 
Sulfanilsäurelösung $(0.5 \mathrm{~g}$ in $150 \mathrm{ccm} 10 \%$ iger Essigsäure) und $1 \mathrm{ccm} a$-Naphthylaminlösung versetzt, dieselbe rote Färbung wie die Zersetzungsflüssigkeit in 10000 facher $\nabla$ erdünnung auf. - Danach enthielt die zersetzte Lösung $3.328 \mathrm{~g} \mathrm{NaNO}_{2}$.

V. $49 \mathrm{~g}$ Kupfer (10 Atome) und $5.4 \mathrm{~g}$ (1 Mol.) Natriumnitrit: $38.0 \mathrm{ccm} \mathrm{NO}, 8.2 \mathrm{~cm} \mathrm{~N} \mathrm{~N}_{2} \mathrm{O}$ in $101.4 \mathrm{ccm}$ Gesamtgas.

$\mathrm{Da}$ das Kupfer viel länger als sonst reduziert worden war, behielt es trotz Wasserbadwärme der Lösung in ihr seine Farbe gröBtenteils bei, zersetzte freilich auch weniger Nitrit, so dab letzteres gröBtenteils unverändert blieb.

$\mathrm{HNO}_{2}$-Bestimmung: a) Auf kolorimetrischem Wege:

Eiue Lösung von $0.1555 \mathrm{~g} \mathrm{NaNO}_{2}$ in $100 \mathrm{l}$ ergab dieselbe Rotfärbung mit Sulfanilsäure und $\alpha$-Naphthylamin, wie die Zersetzangsflüssigkeit in 20000 facher Verdünnung. $\mathrm{NaNO}_{2}$.

Die $160 \mathrm{ccm}$ Zersetzungsflüssigkeit enthielten also $4.960 \mathrm{~g}$

b) Auf maBanalytischem Wege:

1. $10 \mathrm{ccm} 1 / 2-\mathrm{n}-\mathrm{KMnO}_{4}$ wurden von $5.73 \mathrm{ccm}$ Zersetzungs. flüssigkeit entfürbt, so daB also $4.816 \mathrm{~g} \mathrm{NaNO}_{2}$ noch in der Lösung waren.

2. $7.1 \mathrm{ccm} 1 / 2-\mathrm{n}-\mathrm{KMnO}_{4}$ wurden von $4.0 \mathrm{ccm}$ der genannten Lösung entfärbt, woraus man auf 4,899 $\mathrm{g} \mathrm{NaNO}_{2}$ schließen kann.

$$
\mathrm{HNO}_{3} \text { - Bestim mung: }
$$

$30 \mathrm{ccm}$ der zersetzten Lösung lieferten $16.0 \mathrm{ccm} \mathrm{N}$ bei $12^{\circ}$ und $762 \mathrm{~mm}$ nach Stehen über Nacht; also enthielten die $160 \mathrm{ccm} \mathrm{N}$ der mit Kupfer behandelten Lösung $0.3113 \mathrm{~g} \mathrm{NaNO}_{3}$.

VI. Die beiden Kupferspiralen im Gewichte ron $12 \mathrm{~g}$ wurden mit Silber überzogen, indem ich sie in $0.1 \%$ iger Silbernitritlösung in einem Reagenzglase 10 Minuten stehen lieB und danach mit Wasser abspülte. - Darauf warf ich sie in eine 5\% ige Lösung von Natriumnitrit, nachdem ich durch dieselbe $1 / 2{ }^{\text {h }}$ lang Kohlendioxyd geleitet hatte.

Die Gasentwicklung war infolge der Versilberung geringer als sonst und hörte schon nach $2^{\text {h }}$ auf.

$16.8 \mathrm{ccm}$ NO und kein $\mathrm{N}_{2} \mathrm{O}$ in $22.0 \mathrm{ccm}$ Gesamtgas.

VII. Dieselben versilberten Spiralen, dieselbe Gewichtsmenge $\mathrm{NaNO}_{2} ; 38.4 \mathrm{ccm} \mathrm{NO}$ und kein $\mathrm{N}_{2} \mathrm{O}$ in $51.8 \mathrm{ccm}$ Gesamtgas. 


\section{Einwirkung anderer Elomente anf Katriumnitrit.}

Kohle: - - -

\section{Zinn:}

Blei: - - - Das Metall wurde vor seiner Verwendung durch Abschaben von seiner Oxydationsschicht befreit und in kleine Stüeke zerschnitten. Nach dem Versuche, der ohne Gasentwicklung verlief, konnte ich in der Lösung Bleinitrit nachweisen.

Eisen:

a) Elektrolyteisen: - - -

b) Eisenfeilspäne ergaben dieselben Zersetzungsgase wie Kupfer. Diese Erscheinung ist auf die hochmolekularen Karbide zurückzuführen, die das unreine Eisen enthält, und die auch durch Reduktion im Wasserstoffetrome in der Hitze nicht beseitigt werden konnten. - Danach war die Nitritlösung auch schwachgrỉn infolge Bildung von Ferronitrit.

Kobalt: Es setzte geringe Gasentwicklung ein. Die Nitritlösung war danach hellgelb, weil sich Natriumkobaltinitrit gebildet hatte:

$$
\mathrm{Co}_{2}+8 \mathrm{NaNO}_{2}+6 \mathrm{H}_{3} \mathrm{O}=2 \mathrm{CoNa}\left(\mathrm{NO}_{2}\right)_{4}+6 \mathrm{NaOH}+3 \mathrm{H}_{2} ;
$$

denn die Lösung reagierte weder mit kaustischen noch mit kohlensauren Alkalien, wohl aber mit Schwefelwasserstoff und Cyankalium. ganz entsprechend dem Verhalten der Salze des dreiwertigen Kobalts.

Berlin, Technisch-chemisches Institut der Technischen Hochschule. 